\title{
"Two for One", Novel Dual Left Anterior Descending Artery (LAD) Variant: Type XIII
}

James R. Pellegrini ${ }^{1}$, Rezwan Munshi ${ }^{1}$, Alejandro Alvarez Betancourt ${ }^{1}$, Billal Tokhi ${ }^{2}$, Amgad N. Makaryus 3,2

1. Internal Medicine, Nassau University Medical Center, East Meadow, USA 2. Cardiology, Nassau University Medical Center, East Meadow, USA 3. Cardiology, Northwell Health, Manhasset, USA

Corresponding author: Alejandro Alvarez Betancourt, orjandela@gmail.com

\begin{abstract}
Dual left anterior descending artery (LAD) is a rare phenomenon that occurs in less than one percent of the population. To date, 12 variants have been identified. Proper identification of coronary vessels is crucial in emergent situations that require prompt action, such as percutaneous coronary intervention (PCI). We propose that our case highlights a novel 13th (type XIII) variant. We present the case of a 57-yearold African American woman with a past medical history of hypertension, glaucoma, cerebral vascular accident, dyslipidemia who presented to the ED complaining of atypical chest pain for one day duration. Electrocardiography showed normal sinus rhythm at 60 beats per minute (bpm), normal axis, normal intervals, no acute ischemic changes, and an isolated T wave inversion in DIII. Cardiac markers were within normal limits. The patient was started on aspirin $81 \mathrm{mg}$, atorvastatin $40 \mathrm{mg}$, and restarted on amlodipine 5mg. Echocardiography showed a left ventricular ejection fraction (LVEF): 65\%, normal right ventricular size and systolic function, mild mitral valve regurgitation, and mild aortic regurgitation. Computed tomographic (CT) angiography showed a novel subtype of dual LAD, the left circumflex and right coronary arteries were patent. The patient was discharged once stabilized and advised to follow up with cardiology. Dual LAD describes a rare anatomic variant in which two coronary branches, known as short and long LAD arteries, supply the territory normally supplied by the solitary LAD artery. To date, 12 variants of dual LAD, classified by origin and course of the short and long LAD arteries, have been described in the literature. To the best of our knowledge, the current case describes a novel subtype of dual LAD, variant XIII. The LAD originates as usual from the left main coronary artery (LMCA) and initially runs in the anterior interventricular groove for a short course before bifurcating into two long LADs which both leave the interventricular groove and course out to the apex. One of the vessels courses laterally and the other courses medially of the interventricular groove. It is pertinent to identify the coronary vessels accurately before certain interventions are taken. Acknowledgement of this phenomenon can help guide accurate management in the future for patients with this condition.
\end{abstract}

Review began 04/12/2021 Review ended 04/25/2021 Published 04/27/2021

\section{๑) Copyright 2021}

Pellegrini et al. This is an open access article distributed under the terms of the Creative Commons Attribution License CC-BY 4.0., which permits unrestricted use, distribution, and reproduction in any medium, provided the original author and source are credited.
Categories: Cardiology, Internal Medicine, Radiology

Keywords: ct coronary angiography, dual lad, acs, atypical chest pain, anatomical variant, lad variant, cardiac anatomy

\section{Introduction}

Dual left anterior descending artery (LAD) is a rare phenomenon that occurs in less than one percent of the population [1]. To date, 12 variants have been identified [2]. Proper identification of coronary vessels is crucial in emergent situations that require prompt action, such as percutaneous coronary intervention (PCI). We propose that our case highlights a novel 13th (type XIII) variant. We present the case of a middleaged black female who presented to the ED complaining of substernal non-radiating atypical chest pain after witnessing an altercation. Upon admission cardiac imaging revealed a novel subtype of dual LAD. The patient was discharged once stabilized and advised to follow up with cardiology. It is pertinent to identify the coronary vessels accurately before certain interventions are taken. Acknowledgement of this phenomenon can help guide accurate management in the future for patients with this condition.

\section{Case Presentation}

The patient is a 57-year-old African American woman with a past medical history of hypertension, glaucoma, cerebral vascular accident (two times, once in 2014 and once in 2016, with no residual deficits), and dyslipidemia who presented to the emergency department (ED) complaining of atypical chest pain for one day's duration. The patient stated that her chest pain started after she witnessed an altercation. She mentioned the pain was substernal, tight/pressure-like in nature, non-radiating, worse with anxiety, and 6/10 in intensity. Her new pain came in the setting of vertigo (for the past four months), lightheadedness, and difficulty ambulating. She denied any alleviating symptoms and the rest of the review of systems was non-pertinent.

Physical exam was unremarkable, except for mild tenderness to palpation of the chest wall and anxiety. An electrocardiography (ECG) was performed and showed normal sinus rhythm at 60 beats per minute (bpm), 


\section{Cureus}

normal axis, normal intervals, no acute ischemic changes, and an isolated T wave inversion in lead 3 . Vitals in the ED were normal except for elevated blood pressure at 170/84. Cardiac markers were within normal limits. Echocardiography showed a left ventricular ejection fraction of $65 \%$, normal right ventricular size and systolic function, mild mitral valve regurgitation, and mild aortic regurgitation. Coronary computed tomography (CCT) (Figures 1,2) was performed to evaluate coronary artery anatomy and assess plaque burden.

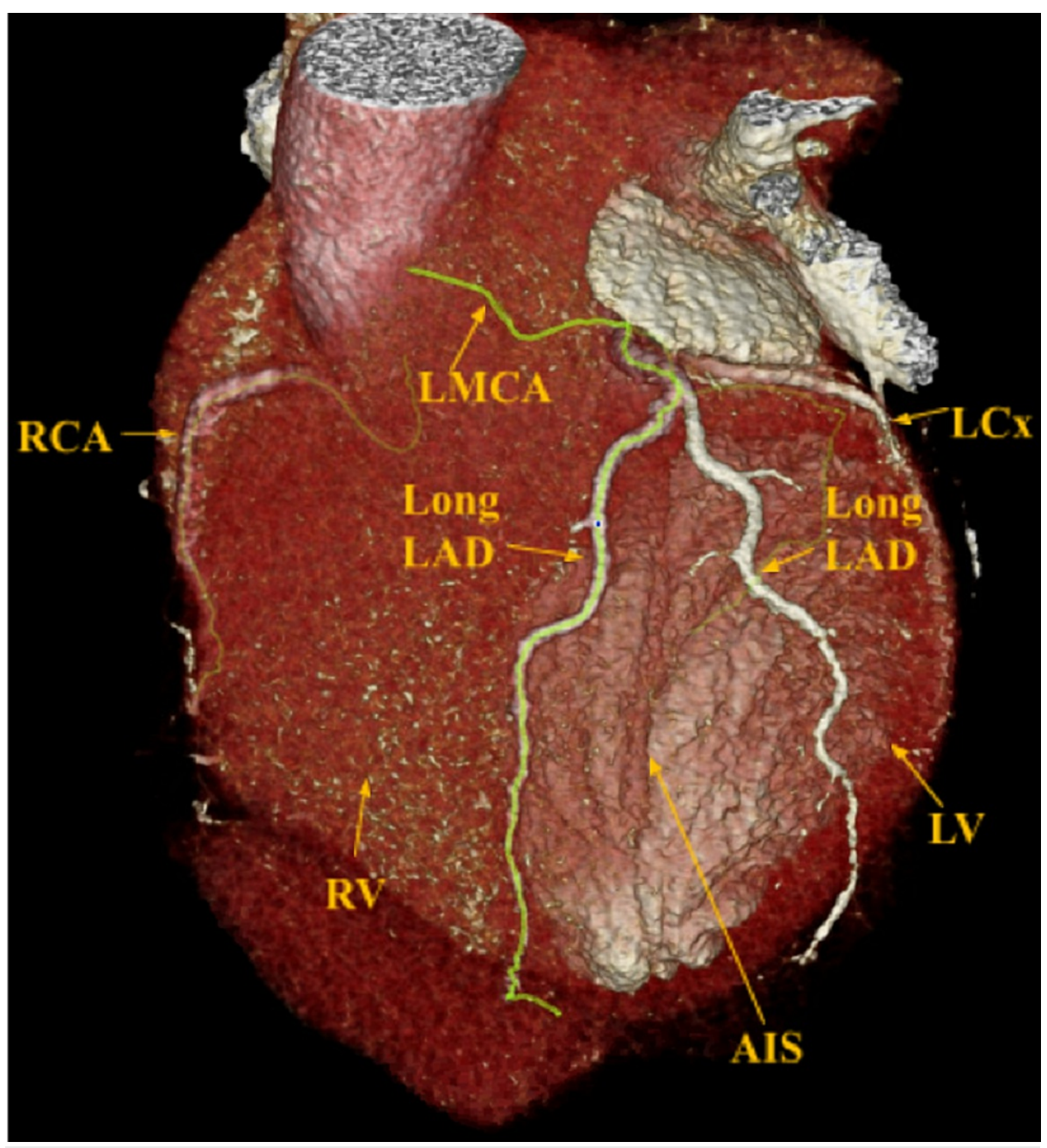

FIGURE 1: CCT showing two long LADs, one coursing laterally and the other coursing medial to the AIS

CCT: Coronary computed tomography; LAD: Left anterior descending artery; AIS: Anterior interventricular sulcus. 


\section{Cureus}

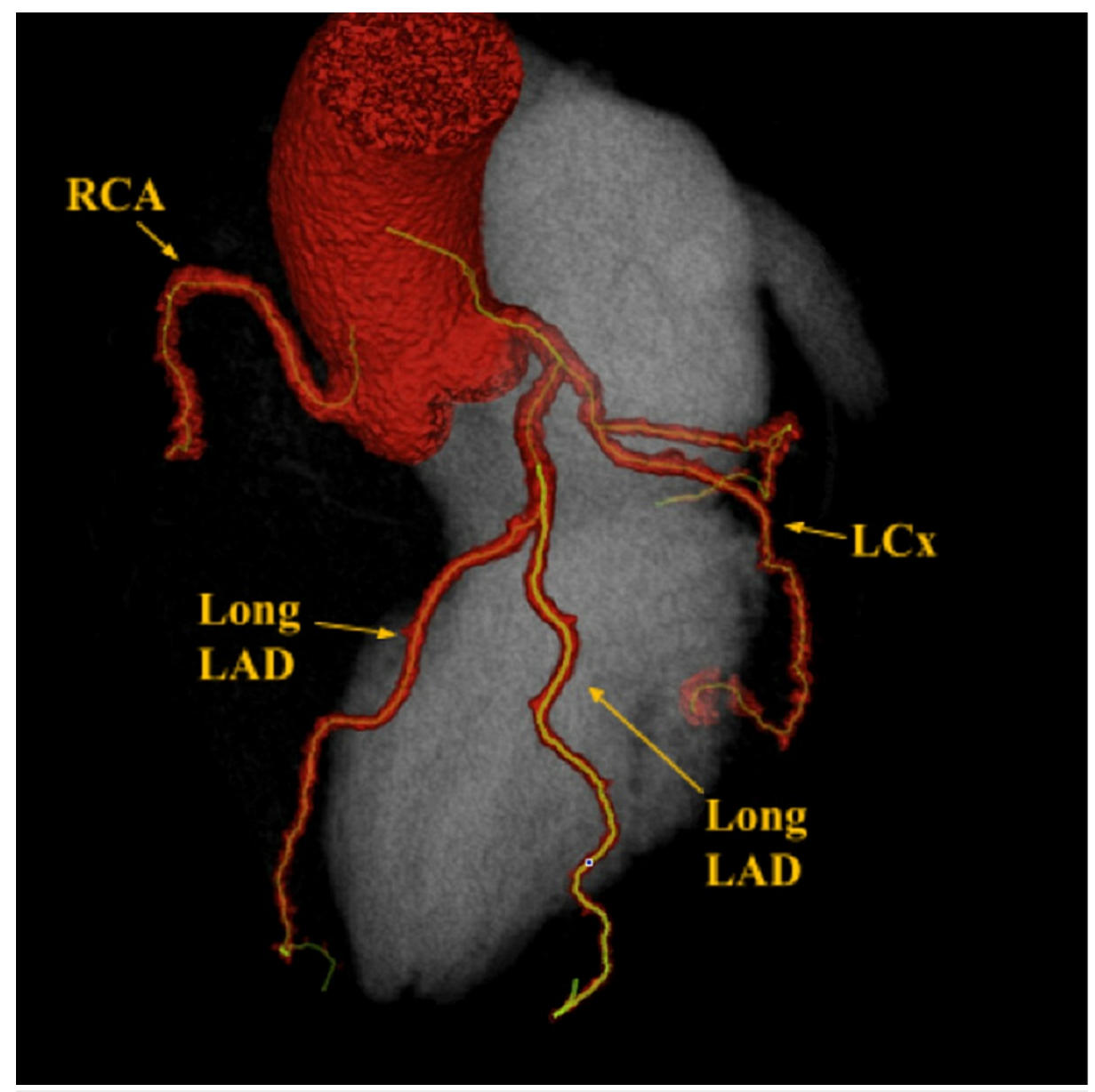

FIGURE 2: CCT showing two long LADs

CCT: Coronary computed tomography; LAD: Left anterior descending artery.

\section{Discussion}

Differential diagnosis included acute coronary syndrome (ACS), musculoskeletal pain, and anxiety-induced chest pain. ACS was ruled out after cardiac markers were negative twice and ECG had no acute ischemic changes. Incidentally, the CCT results showed a left anterior descending artery (LAD) that originates from the left main coronary artery (LMCA) and initially runs in the anterior interventricular sulcus (AIS) for a short course before bifurcating into two long LADs which both leave the AIS and course out to supply the apex. While it may be considered that the second long LAD may represent a large first diagonal branch; that would assume that the patient has an LAD that resides in the expected anatomic position in the anterior interventricular groove, but in fact it does not, and instead another Long LAD, that runs medially to supply the septum and medial structures, is noted and does not run in the anterior interventricular groove [3]. One of the vessels was noted to course laterally and the other coursed medially of the AIS. The left circumflex (LCx) and right coronary arteries (RCA) were patent (Figures 1, 2).

The patient was continued on her home medications for hypertension - amlodipine $5 \mathrm{mg}$ oral daily. She was discharged once stabilized and advised to follow up as an outpatient. While dual LAD is rare (Sidhu and Wander found $0.68 \%$ prevalence for any type of dual LAD) and presumed benign, it presents various diagnoses and treatment challenges of which practitioners must be aware [4-6]. Prerequisite to successful surgical intervention, like revascularization, is the precise knowledge of the anatomical features to be evaluated. For example, if both the short and long LAD arteries are significantly stenosed in a patient, revascularization of both vessels may be necessary to restore blood supply to the septum and anterior wall. Additionally, as CCT has become the noninvasive imaging modality of choice for the diagnosis of cardiovascular coronary pathology, it is particularly imperative that clinicians be aware of these variants to allow for precise evaluation and management.

Typically, the LMCA gives rise to the LAD artery, which descends through the epicardial fat of the AIS. It then proceeds to give off septal and diagonal branches which supply the anteroseptal and anteroapical regions of the heart [1]. Dual LAD describes a rare anatomic variant in which two coronary branches, known as the "short" and "long" LAD arteries, supply the territory normally supplied by the solitary LAD artery. 
Dual LAD was first reviewed and classified by Spindola-Franco et al. in 1983 when just three variants had been discovered [2]. To date, 12 variants of dual LAD, classified by origin and course of the short and long LAD arteries, have been described in the literature. Retrospective analyses have revealed that Type I dual $\mathrm{LAD}$ (in which the short LAD artery originates from the LAD proper and terminates in the AIS, while the long LAD artery originates from the LAD proper and descends to the left of the AIS before entering the AIS more distally) is the most common variant. Current classification of dual LAD can be seen in Table 1 .

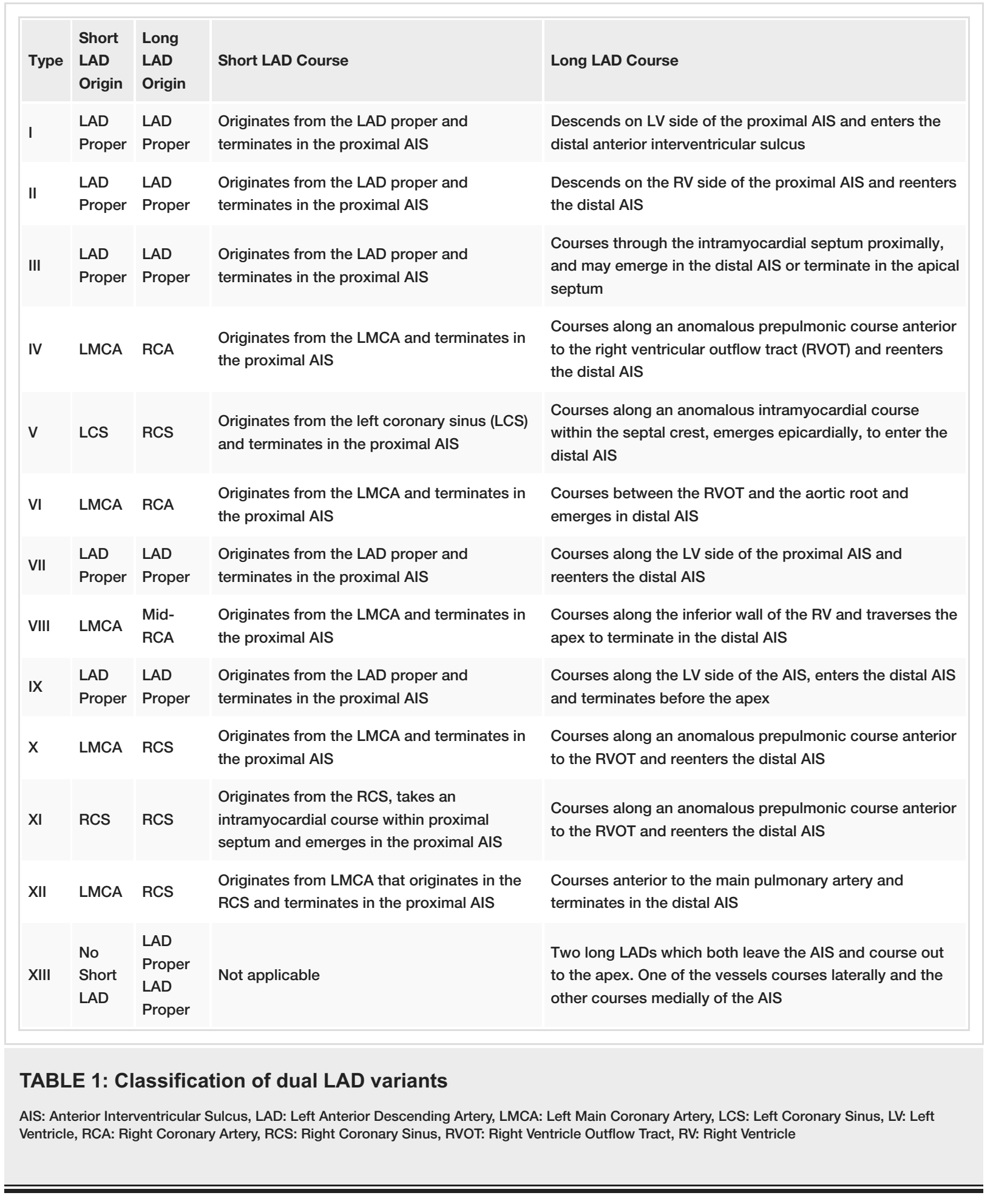

\section{Conclusions}

To the best of our knowledge, the current case describes a novel subtype of dual LAD, which we propose to name variant XIII. In theory, mortality associated with LAD occlusion should be minimized if there are two LADs supplying the same territory as a single LAD. However, in patients with long-standing atherosclerosis, it is possible that both LADs may be occluded. Therefore, it is pertinent to identify the coronary vessels accurately before certain interventions are taken. Our case proposes a novel variant (Type XIII) of the dual 
LAD anatomic phenomenon never before described in the world literature. Acknowledgement of this phenomenon can help guide accurate management in the future for patients with this variant.

\section{Additional Information}

\section{Disclosures}

Human subjects: Consent was obtained or waived by all participants in this study. Conflicts of interest: In compliance with the ICMJE uniform disclosure form, all authors declare the following: Payment/services info: All authors have declared that no financial support was received from any organization for the submitted work. Financial relationships: All authors have declared that they have no financial relationships at present or within the previous three years with any organizations that might have an interest in the submitted work. Other relationships: All authors have declared that there are no other relationships or activities that could appear to have influenced the submitted work.

\section{References}

1. Malagò R, Pezzato A, Barbiani C, Alfonsi U, Nicolì L, Caliari G, Pozzi Mucelli R: Coronary artery anatomy and variants. Pediatr Radiol. 2011, 41:1505-1515. 10.1007/s00247-011-2218-9

2. Spindola-Franco H, Grose R, Solomon N: Dual left anterior descending coronary artery: angiographic description of important variants and surgical implications. Am Heart J. 1983, 105:445-455. 10.1016/00028703(83)90363-0

3. Pandey NN, Shaw M, Sharma A, Ganga KP, Gulati GS: Yet another novel variant of dual left anterior descending artery: type XII. Heart Lung Circ. 2020, 29:33-35. 10.1016/j.hlc.2019.07.022

4. Şeker M: Prevalence and morphologic features of dual left anterior descending artery subtypes in coronary CT angiography. Radiol Med. 2020, 125:247-256. 10.1007/s11547-019-01124-7

5. Bozlar U, Uğurel MŞ, Sarı S, Akgün V, Örs F, Taşar M: Prevalence of dual left anterior descending artery variations in CT angiography. Diagn Interv Radiol. 2015, 21:34-41. 10.5152/dir.2014.14275

6. Sidhu NS, Wander GS: Prevalence and characteristics of dual left anterior descending artery in adult patients undergoing coronary angiography. Future Cardiol. 2019, 15:425-435. 10.2217/fca-2019-0052 